\title{
Análisis del posicionamiento de las marcas de equipos hospitalarias. Caso de estudio empresa Bioinnova S.A.S.
}

\author{
Analysis of hospitals equipment brand positioning case of study Bioinnova S.A.S. COMPANY
}

Recibido: 30-06-2020 • Aprobado: 06-04-2021 • Página inicial: 81 - Página final: 105

\author{
Lina María Arango* \\ Eduardo Londoño Montoya**
}

\begin{abstract}
Resumen: se presenta el análisis de las estrategias de posicionamiento y el estudio de la marca realizado a la empresa colombiana Bioinnova S.A.S., especializada en la fabricación de equipos médicos que desea ampliar su cobertura internacional. El objetivo estuvo direccionado al posicionamiento que tiene la marca en Chile, Ecuador y Perú. La metodología utilizada fue descriptiva, de corte cualitativo. En los resultados obtenidos, se encuentra que se deben realizar cambios organizacionales al interior de la empresa, con el fin de liderar el proceso de comercio exterior. Se decide posicionar la marca, inicialmente en Chile, gracias a la estabilidad y fortaleza de todos los medidores macroeconómicos, al crecimiento de la inversión extranjera directa, a las perspectivas de crecimiento y al alto poder adquisitivo de sus ciudadanos. Además, este país invierte alrededor del 7,2\% del producto interno bruto en salud, aportando valor comercial para la empresa analizada.
\end{abstract}

Palabras clave: columna médica; investigación de mercados; panel médico; posicionamiento y registro de la marca.

\begin{abstract}
The analysis of the positioning strategies and brand study carried out at the Colombian company Bioinnova S.A.S, specialized in the manufacture of medical equipment that wishes to expand its international coverage, is presented. The objective was directed to the study of the positioning that the brand has in Chile, Ecuador and Peru. The methodology used was descriptive, qualitative. In the results obtained, it is found that the company must make organizational changes within the company, in order to lead the foreign trade process and it is decided to position the brand initially in Chile, because they have stability and strength from all macroeconomic measures, the growth of foreign direct investment, growth prospects and the high purchasing power of its citizens. In addition, this country invests around $7,2 \%$ of the gross domestic product in health, providing commercial value for the analyzed company.
\end{abstract}

Keywords: Medical power column; market research; medical headwall; brand positioning; trade mark.

JEL: R9

\footnotetext{
* Tecnóloga en Mercadeo Internacional, Administradora de Empresas, Especialista en Gerencia de Servicios de Salud y Magíster en Administración. Administradora BIOINNOVA S.A.S

linarango2008@hotmail.com; administracion@bioinnova.com.co

ORCID: https://orcid.org/0000-0001-5614-8985

** Ingeniero Civil, Administrador de Empresas, Especialista en Logística, Especialista en Gerencia y Magíster en Administración, Integrante de la línea Comercio Exterior y Negociación Internacional del Grupo ECOSOL de la Universidad Católica Luis Amigó.

eduardo.londomo@amigo.edu.co

ORCID: https://orcid.org/0000-0003-1469-3322
} 


\section{Análise do posicionamento de marcas de equipamentos hospitalares. Estudo de caso da empresa Bioinnova S.A.S.}

Resumo: apresenta-se uma análise das estratégias de posicionamento e o estudo da marca realizado para a empresa colombiana Bioinnova S.A.S, especializada na fabricação de equipamentos médicos que deseja expandir sua cobertura internacional. O objetivo foi direcionado ao posicionamento da marca no Chile, Equador e Peru. A metodologia utilizada foi descritiva e de característica qualitativa. Nos resultados obtidos, constata-se que mudanças organizacionais devem ser feitas dentro da empresa, a fim de conduzir o processo de comércio exterior. Decidiuse posicionar a marca, inicialmente no Chile, graças à estabilidade e robustez de todas as medidas macroeconômicas, ao crescimento do Investimento Estrangeiro Direto, às perspectivas de crescimento e ao alto poder aquisitivo de seus cidadãos. Além disso, este país investe cerca de 7,2\% do Produto Interno Bruto em saúde, fornecendo valor comercial para a empresa analisada.

Palavras-chave: coluna médica; investigação de mercados; painel médico; posicionamento e registro de marca. 


\section{Introducción}

La investigación busca el diseño de estrategias de posicionamiento de la marca de la empresa Bioinnova S.A.S., para la comercialización de equipos para la infraestructura hospitalaria, se analizan los mercados de Ecuador, Perú y Chile.

Se pretende que la investigación pueda servir como un vínculo real de acercamiento entre universidad-empresa, en el que se confronten teorías con la práctica y se compartan experiencias, para obtener unos resultados que contribuyan a la inserción y posicionamiento de las pymes nacionales en los mercados internacionales de una forma sólida y organizada, que garantice la permanencia en ellos. El posicionamiento de la marca, le permitirá a la empresa aparecer como una organización confiable con un alto reconocimiento en estos mercados internacionales.

La investigación se llevó a cabo por un interés manifiesto de los socios de la empresa, razón por la cual se piensa en dos aspectos cruciales del presente trabajo: por un lado, las limitaciones de la investigación obedecerán únicamente a los tiempos dentro de los cuales se desarrolle la investigación; por otro lado, la viabilidad del trabajo se facilita puesto que cuenta con el apoyo de la gerencia de la empresa, que tiene un compromiso con los aspectos de reorganización y de mejoramiento continuo, que permitan a la empresa posicionarse y mantenerse en el punto al que se pretende llegar en el ámbito internacional.

Bioinnova S.A.S. pertenece al sector metalmecánico, dedicada al diseño, fabricación, distribución e instalación de equipos para infraestructura hospitalaria; estos equipos son funcionales, confiables y seguros para los pacientes. Para garantizar la calidad de sus productos, la empresa cuenta con recursos humanos y técnicos especializados, innovando procesos y vigilando que la relación costobeneficio sea acorde al mercado, la empresa cuenta con la certificación UL, emitida por Underwriters Laboratories, símbolo de mayor reconocimiento para productos que cumplen con los estándares de seguridad y calidad de productos fabricados en los Estados Unidos y Canadá, característica fundamental para competir en mercados internacionales. A su vez, la empresa ha iniciado operaciones de comercialización de sus productos en el exterior, en países como Perú, Chile y Costa Rica; esto ha llevado a desarrollar un proceso de posicionamiento de la marca en estos países y, por esa vía, a aumentar el volumen de operaciones internacionales de la empresa; se incluye Ecuador debido a que en este país no existen empresas dedicadas a la fabricación de equipos para infraestructura hospitalaria. 


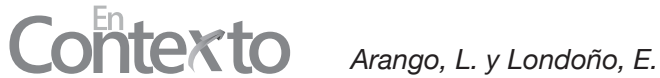

Por ello, se plantea una adecuada gestión de una marca que se traduce en la creación de valor para los consumidores, es decir, a través del manejo de la marca se supera la diferencia entre la funcionalidad del producto y los atributos simbólicos y sociales del mismo (Ortegón, 2014).

La comercialización de productos y/o servicios presenta obstáculos debido a que el comprador suele confundirse con la selección del mismo, dada la diversidad de marcas que aparecen en productos y/o servicios de la misma categoría. Además, las mayores exigencias y el aumento de costos de los procesos de producción, permite a la gestión de la marca crear espacios a las organizaciones, en los cuales obtienen beneficios económicos a través del reconocimiento de los bienes en el consumidor, esto es, las marcas ya no aparecen como simples herramientas de mercadeo y han pasado a ser elementos intangibles sobre los que las empresas construyen relaciones a largo plazo con sus clientes (Medina-Aguerrebere, 2014).

Dada la importancia de generar un impacto de marca, se llega a la necesidad de desarrollar una investigación de mercados, esto con el fin de vincular al consumidor con el producto que diseña, fabrica y comercializa y se han estableciendo las herramientas de marketing que se debe utilizar para ingresar al mercado suramericano frente a bienes específicos, como son los equipos para infraestructura hospitalaria.

Por lo tanto, la investigación de mercados se presenta como una herramienta para el seguimiento, la recolección y el análisis sistemático de toda la información, cuyo objetivo se centra en la toma de decisiones estratégicas más acertada, en cuanto a la comprensión del entorno del consumidor y la evaluación de los posibles competidores para determinar las oportunidades y amenazas del entorno (Kotler, 2017).

Las operaciones de carácter internacional, que ha realizado la empresa hasta el momento, podrían considerarse como casuales, puesto que se presentan de forma esporádica y no obedecen a estrategias consideradas en programas de mercadeo, sino que se han dado únicamente fundamentadas en la calidad del producto y en la escasez de oferentes de esta línea de productos hospitalarios. Así, la empresa analizada, decide ser pionera en la comercialización de sus productos, a través de un posicionamiento de la marca que le permita aparecer como marca exclusiva de la fabricación, comercialización e instalación de equipos para infraestructura hospitalaria. 


\section{Antecedentes}

La empresa Bioinnova S.A.S. es una empresa del sector metalmecánico dedicada a la arquitectura hospitalaria y a la prestación de servicios industriales, está en capacidad de producir mercancías certificadas; soportada en sus altos estándares de calidad, aunque la comercialización de ellas obedece más a negociaciones basadas en la misma calidad de los productos y no a verdaderos procesos de mercadeo internacional, estandarizados, correctamente planeados y desarrollados como debería ser. Cuenta con cuatro años en el mercado y está dedicada al diseño, fabricación, distribución e instalación de equipos para infraestructura hospitalaria. Es una pyme con reconocimiento nacional, ha distribuido sus productos en las principales clínicas de la ciudad y fuera de ella.

Mediante la publicidad voz a voz a nivel nacional, se han distribuido los productos en clínicas de tercer nivel como: Clínica la Misericordia-Barranquilla, Clínica Avidanti-Santa Marta, Clínica la Magdalena-Barrancabermeja, Eusalud-Bogotá, Nuevo hospital de Zipaquirá-Zipaquirá, Nuevo hospital de Tocancipá-Tocancipá, Instituto Cancerológico-Bogotá, entre otros. Basado en los resultados de ventas nacionales, Bioinnova S.A.S. ve una oportunidad de negocio y decide exportar sus productos, obteniendo una excelente respuesta. En 2020, la empresa cuenta con clientes en Ecuador, Estados Unidos, Chile, Costa Rica y Perú.

\section{Marco teórico}

Una marca es un signo distintivo que indica que ciertos bienes o servicios han sido producidos o proporcionados por una persona o empresa determinada. Su origen se remonta a la antigüedad, cuando los artesanos reproducían sus firmas o "marcas" en sus productos utilitarios o artísticos. A lo largo de los años, estas marcas han evolucionado hasta configurar el actual sistema de registro y protección de marcas. En la diferenciación del producto, los consumidores del mundo están expuestos a miles de marcas que se le presentan a través de múltiples medios de comunicación, tratando de llegar a ellos para que queden con la imagen y el mensaje que se le quiere transmitir. Para conseguir esto, un aspecto se toma para la diferenciación: ser diferente a los otros es la clave para destacar un producto frente a los demás de su clase, se trata de conseguir mostrar algo que haga único el producto ante los ojos del consumidor (Godás, 2006). La diferenciación se define como la distinción entre productos que cumplen el mismo propósito, pero fueron hechos por diferentes productores y, además, compiten entre ellos (Kotler, 1994). 


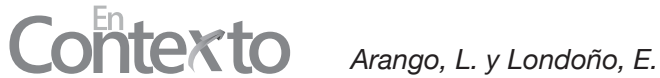

De esta manera, esta definición adopta un enfoque que incluye todos los aspectos de la oferta $y$, además, asegura que la diferenciación significa la introducción de características diferenciales, bien sea de calidad, estilo o imagen de la marca, como base para ser líder en el mercado; adicionalmente, dichas características tendrán que cumplir con ciertos requisitos: ser percibidas por los consumidores, no presentar dudas respecto a posibles imitaciones de la competencia y un valor real para el proveedor.

La diferenciación es una estrategia de marketing que trata de resaltar las características del producto, sustanciales o accesorias, que pueden contribuir a que se perciba como único, para así crear una situación de monopolio en el mercado, pero esta diferenciación contendrá atributos esperados por el consumidor, y atributos complementarios de los productos (Kotler, 1994).

Posicionamiento de la marca: es un tipo de signo distintivo que pone una identificación muy específica a los productos o servicios de una empresa o empresario. También permite a los consumidores identificar un producto o servicio y recordarlo de forma que puedan diferenciarlo de uno igual o semejante, ofrecido por otro empresario.

Para la Asociación Americana de Marketing, marca es un nombre, un término, una señal, un símbolo, un diseño o una combinación de alguno de ellos que identifica productos y servicios de una empresa y lo diferencia de los competidores. Aunque la marca no es un mero nombre y un símbolo, se ha convertido en una herramienta estratégica dentro del entorno económico actual.

El posicionamiento es aquello que una marca está dispuesta a hacer para ser quien quiere ser; delimita el espacio que ocupará una marca en sus mercados. Esto significa que es una herramienta fundamental que determina quién es, qué hace y por qué es una marca importante para su mercado. Asimismo, determina el significado y la forma en la que se va a competir en el mercado. Consecuentemente, el posicionamiento de la marca señala qué espacio ocupa la marca en la mente de los consumidores. A su vez, el posicionamiento de la marca favorece la generación de vínculos emocionales entre la marca y la mente de sus consumidores; el motivo puede ser atribuido posiblemente a la falta de una clara base teórica o a la relativa sencillez con que el concepto es utilizado por los agentes o usuarios del marketing. Así, para reafirmar el significado de posicionamiento, usualmente se recurre a una serie de términos complementarios relacionados, tales como "posición del producto", "posición", "posicionamiento de producto" o "posicionamiento de mercado", que, incluso, presentan algún cruce conceptual o una superposición de los conceptos y aplicaciones. 
Se entiende que un objeto está posicionado cuando puede ser identificado en un lugar en comparación con los lugares que otros objetos ocupan. La relevancia del concepto de categoría de producto es explícita en los conceptos de posicionamiento. Generalmente, los profesionales de marketing asumen que los clientes agrupan los productos de forma jerárquica en niveles variados de especificidad. Para determinar el posicionamiento de la marca se debe desarrollar todo un compromiso con la cultura, procesos, comportamientos, empleados y la comunicación de una marca (Serralvo y Tadeu, 2005).

La investigación de mercados: la globalización de los mercados y la creciente interrelación e interdependencia de las diferentes economías han acelerado en gran medida los procesos de internacionalización de las empresas. En este contexto, la exportación se presenta como la vía más utilizada y tradicional que utilizan las empresas para su entrada en los mercados foráneos, configurándose como la opción estratégica más efectiva para asegurar la supervivencia y el crecimiento de las organizaciones que han optado por iniciar sus propios procesos de internacionalización. Las empresas ven en las exportaciones el medio directo para hacer frente a la cada vez mayor competencia extranjera en sus mercados domésticos, así como a la forma de ampliar su cobertura en el mercado y, por esta vía, aumentar su rentabilidad. Mas las exportaciones como tal no son un fin en sí mismas, puesto que el objetivo de las empresas exportadoras será obtener ventajas competitivas en los países mercados donde pretendan competir (Delgado-Márquez et al., 2013).

Pero esto dependerá de un diseño de estrategias eficientes y eficaces de mercadeo que le den a la organización la oportunidad de adaptarse a las condiciones de los mercados extranjeros para conseguir una mejora continua del resultado exportador, con base en los recursos y capacidades disponibles. Será solamente a partir del conocimiento de todas las diferencias de tipo económico, cultural y social, entre su mercado nacional y los mercados foráneos, que se podrá generar inteligencia de mercados, y así poder procesar toda la información vital y relevante acerca de los clientes, la competencia y los proveedores, entre otros, para ponerla a disposición de toda la organización y entrar en un proceso de toma de decisiones estratégicas, plenamente fundamentadas, en cuanto a la actividad exportadora.

Dice Review (2000) que la inteligencia de mercados no es más que el resultado de la convergencia de la evolución de la inteligencia competitiva con la emergencia del mercadeo como un área estratégica de la empresa. Así, la inteligencia de mercados abarca todas las principales actividades de las empresas privadas e incluye también las organizaciones públicas. 


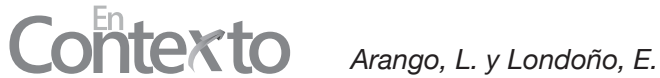

Estructura organizacional: las estructuras organizacionales traen consigo consideraciones mucho más profundas e incluyentes que la simple representación gráfica conocida como organigrama, con la cual se tiende a confundir la estructuración, involucra todo un proceso que inicia con la planeación de estrategias, para terminar con el desarrollo del objeto social de la organización. La estructura de la organización debe diseñarse de forma que haya claridad total acerca de quién debe realizar unas tareas específicas y quién será el responsable por sus resultados. De esta forma, se evitan los problemas originados en la imprecisión de la asignación de responsabilidades y se consigue una toma de decisiones adecuada con los objetivos de la empresa.

Esto significa que la estructura es una de las herramientas de las que se vale la organización para conseguir sus objetivos con eficacia. Por esta razón es que la estructura comprende la forma en que se dividen, agrupan y coordinan las actividades en una organización, así como las relaciones entre los gerentes y los empleados.

El análisis de la estructura organizacional empresarial deberá enmarcarse en la Teoría de la Contingencia Estructural, la cual surge para mostrar, a partir de una larga serie de investigaciones, que no existe una única forma de estructuración; esto se debe a que la división del trabajo y su coordinación dependerán de múltiples factores, entre ellos, las condiciones situacionales en las cuales la organización opera. Las diferentes investigaciones acerca de la estructura organizacional, desde sus múltiples aproximaciones, han tratado de comprender su integración y coordinación para explicar el fenómeno de la división del trabajo.

La Teoría de la Contingencia concibe que las organizaciones funcionan como sistemas abiertos, cuya supervivencia se halla sujeta al logro de un delicado equilibrio dinámico interno-externo. Según este enfoque, la relación entre la organización y su entorno inmediato es únicamente de carácter adaptativo; de ello se desprende que el modelo óptimo de organización está sujeto al entorno con el que se relaciona (Zapata, 2019).

\section{Metodología}

Se realizó un análisis descriptivo de tipo cualitativo, el cual se desarrolló en tres fases:

1. Recolección de información con los clientes internacionales de la empresa, se eligieron organizaciones pertenecientes al sector salud, para recopilar la información primaria. Se aplicó una encuesta, a través de la interface Google Forms, a 10 empresas que fueran clientes activos, las características 
predominantes para la selección es que fueran clínicas de tercer nivel de los países de Chile, Costa Rica, Estados Unidos, Ecuador y Perú; a su vez, que tuvieran la necesidad de adquirir los productos de la empresa analizada, estas clínicas tienen la capacidad de atender problemas que no pueden ser resueltos en los otros dos niveles y que requieren un enfoque especializado o una tecnología específica; en términos generales, costosa.

2. Se realizó un análisis del mercado, la empresa tiene como meta tres países suramericanos y, con los resultados de la primera fase, se proyecta el posicionamiento a realizar en el país elegido, estos estuvieron compuestos por impresiones, opiniones y perspectivas de los investigadores, basados en las cinco fuerzas de Porter.

3. Consistió en recopilar información secundaria para poder tomar decisiones gerenciales frente a la incursión en mercados internacionales, aplicando un análisis PEST en los países elegidos.

La investigación se apoyó en fuentes de información secundarias, contenidas en libros, revistas especializadas, investigaciones sobre el tema trabajos de grado, anuarios e información de internet. Se acudió a bases de datos tales como: Country Watch, Scielo, Redalyc, Dialnet, Legiscomex, Scopus y Google Scholar y páginas oficiales de la Superintendencia de Industria y Comercio, el Banco Mundial y las Cancillerías de los países analizados.

\section{Resultados}

La recolección de la información se planeó en tres fases.

En la primera fase, se realizó la recolección de información primaria; el instrumento fue aplicado a través de una encuesta realizada entre septiembre y noviembre de 2019, cuyo índice de satisfacción con los clientes internacionales fue superior al $80 \%$ en una escala de 1 a 100; cada pregunta contó con cuatro niveles de evaluación: Muy bueno (76-100), bueno (56-75), regular (26-55) y malo (0-25). 


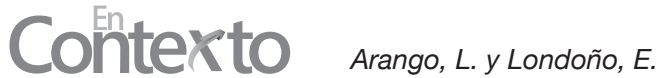

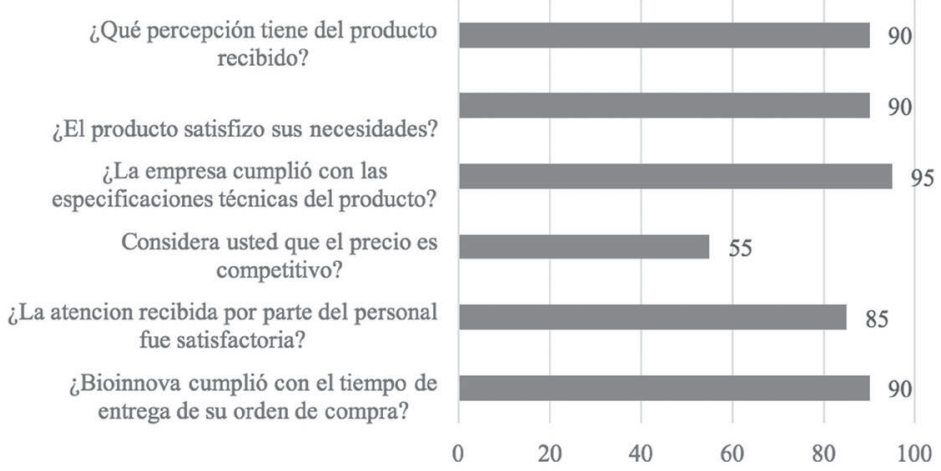

Figura 1. Encuesta de satisfacción

Elaboración propia, 2019.

La empresa cumple con las especificaciones técnicas requeridas por los clientes internacionales, ofreciendo calidad, diseño exclusivo, cumplimiento con fechas de entrega, productos con estética acorde a la infraestructura hospitalaria, productos funcionalmente confiables y seguros para los pacientes y relación costo-beneficio, puntos fundamentales para establecer una buena relación entre las partes interesadas. Respecto al tiempo de entrega de una orden de compra, varía de acuerdo con el diseño, la cantidad de productos solicitados y los procesos que se tengan que utilizar para el desarrollo de los mismos. Se precisa que no todos los procesos se hacen directamente en la empresa; por tal motivo, se deben establecer tiempos de acuerdo con la programación de los proveedores.

Para el desarrollo de un producto existen los siguientes pasos:

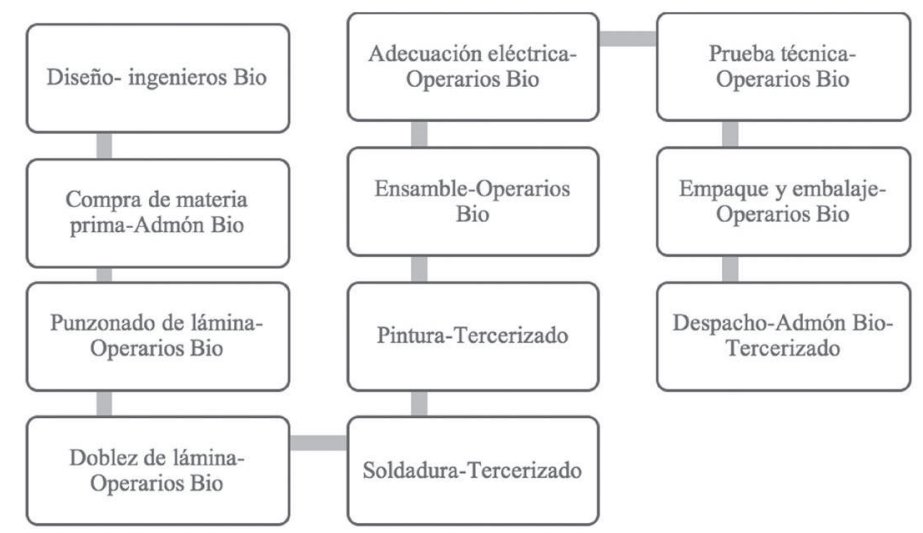

Figura 2. Procedimiento de producción

Tomado de Arango (2019). 
Estos procesos pueden tardar entre 20 y 40 días hábiles según las necesidades y condiciones previamente mencionadas.

Las respuestas de los clientes dieron como resultado un $90 \%$ de favorabilidad por la experiencia obtenida, indicando que Bioinnova S.A.S. está cumpliendo con las fechas que fueron asignadas desde la recepción de la orden de compra hasta la entrega de la misma.

Otro aspecto analizado fue el nivel de percepción frente al servicio al cliente que se ofrece en la empresa; con ese fin, los clientes internacionales tienen un primer encuentro con el gerente de la compañía con quien establecen una relación permanente durante todo el proceso, esta persona es la encargada de la negociación tanto económica, como de coordinar los tiempos de entrega. Después de tener las condiciones del negocio claras, la administración de la empresa se encarga de informar al cliente las novedades que se puedan presentar en el proceso y coordina la operación internacional.

El operador logístico es el encargado de entregar la mercancía al cliente en excelentes condiciones. Estos operadores son 100\% confiables cuando todo el proceso es realizado y contratado directamente por la empresa, dado que existen condiciones específicas para ser proveedor de la compañía: cumplimiento de acuerdos de seguridad y confidencialidad, inspecciones físicas, estudio de análisis de riesgos de acuerdo con la matriz que fue diseñada por el sistema de gestión de calidad de la empresa.

Cuando las operaciones internacionales son realizadas por el cliente, Bioinnova S.A.S. asume la responsabilidad única y exclusivamente hasta el momento de la entrega del producto a dicho operador. Los clientes a esta pregunta calificaron la atención recibida en un $98 \%$ de satisfacción, resultado que, para la empresa analizada, significa una excelente comunicación entre los directivos y un gran logro dado que se ha sobrepasado barreras culturales y acervos legales que generan buenas relaciones comerciales.

Otro de los cuestionamientos realizados estuvo enfocado en el precio, estos fueron fijados después de hacer un análisis de costos y ofertas en el mercado; en esta encuesta, la calificación de los clientes fue de un 55\% de favorabilidad, siendo suficiente para la empresa.

A nivel interno, nunca se ha pretendido que la empresa sea reconocida en el mercado por precios bajos o iguales a los de la competencia. La compañía busca reconocimiento por calidad y exclusividad. Sin embargo, se puede considerar el $45 \%$ restante como una oportunidad para minimizar costos y aumentar productividad. A 


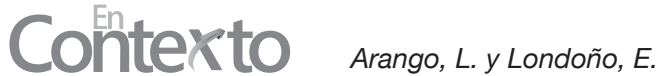

raíz de esto, se les cuestiona a los clientes si el producto satisfizo sus necesidades; cada uno de los productos que son diseñados, fabricados y comercializados por la empresa tienen una promesa de valor: "satisfacer las necesidades del cliente" y se está cumpliendo con este objetivo; según el resultado de la encuesta, el 90\% de satisfacción, demuestra que el cliente está a gusto con el producto recibido.

Un punto importante de este cuestionario se enfocó en las especificaciones técnicas del producto, este interrogante hace recordar que la misión de la empresa es diseñar, fabricar, distribuir e instalar equipos para la infraestructura hospitalaria, funcionalmente confiables y seguros para los pacientes, garantizando el cumplimiento de las especificaciones técnicas del producto, según las necesidades del cliente. Por ello, es de suma importancia el 95\% de satisfacción que se refleja en la encuesta realizada a los clientes internacionales, porque demuestra que se está cumpliendo con el objetivo principal de la compañía.

Se planteó el diseño de estrategias de posicionamiento de la marca de la empresa Bioinnova S.A.S. para la comercialización de equipos para la infraestructura hospitalaria en Chile, Ecuador y Perú. Para la llegada de la marca empresarial, a los mercados internacionales se propuso una estrategia de diferenciación del producto, aprovechando las dos ventajas competitivas que han sido resaltadas por sus clientes, calidad y el servicio, incluso son consideradas más relevantes, sobre los precios ofrecidos por la empresa.

En la segunda fase, se realizó el análisis del mercado. El análisis fue realizado en Chile, Perú y Ecuador, que son los tres países en donde la empresa se proyecta internacionalmente.

Tabla 1

Análisis de mercado

\begin{tabular}{|c|c|c|c|}
\hline $\begin{array}{l}\text { Tipo de } \\
\text { clientes }\end{array}$ & Clientes & Requerimiento de los clientes & $\begin{array}{l}\text { Requerimiento interno Bioinnova } \\
\text { S.A.S. }\end{array}$ \\
\hline $\begin{array}{l}\text { Distribui- } \\
\text { dores }\end{array}$ & $\begin{array}{l}\text { Cliente Chile: } \\
\text { Distribuidor 01. } \\
\text { Diseño, ingeniería } \\
\text { y construcción. } \\
\text { Cliente Perú: } \\
\text { Distribuidor 02. }\end{array}$ & $\begin{array}{l}\text { Calidad, diseño exclusivo, cum- } \\
\text { plimiento con fechas de entrega, } \\
\text { productos con estética acorde a } \\
\text { la infraestructura hospitalaria } \\
\text { del cliente, productos funcio- } \\
\text { nalmente confiables y seguros } \\
\text { para los pacientes; relación cos- } \\
\text { to-beneficio. Cumplimiento de } \\
\text { contratos, confidencialidad. }\end{array}$ & $\begin{array}{l}\text { Control de calidad durante todo el } \\
\text { proceso de producción y al producto } \\
\text { terminado y realizado por el auxiliar } \\
\text { de planta. } \\
\text { Recibo de satisfacción del producto, a } \\
\text { través de la firma y sello de la factura. } \\
\text { Aceptación de la guía de transporte, } \\
\text { cuando el producto sea despachado } \\
\text { por este medio. } \\
\text { Encuesta de satisfacción de clientes. }\end{array}$ \\
\hline
\end{tabular}

Tomado de Arango (2019). 
La atención para los clientes y el cumpliendo con las exigencias (descritas en el cuadro anterior) van a requerir de la creación de un nuevo cargo dentro de la empresa, tal como se planteó en los objetivos específicos. Este cargo a proveer será el de director de mercadeo, cuya función principal será la consecución de nuevos clientes y el mantenimiento de los clientes actuales; igualmente, de la exploración de posibles nuevos mercados internacionales, dentro de los que se han identificado como potenciales: Costa Rica, México y Estados Unidos. Las exigencias de los clientes están identificadas por la empresa, razón por la cual cumplir con estos requisitos no solo satisface las necesidades y expectativas de los mismos, sino que es la garantía para permanecer vigente en el mercado y ser un referente importante para nuevos clientes. Se presenta el análisis de la competencia en los países seleccionados, Chile, Ecuador y Perú.

Tabla 2

Análisis del mercado competitivo

\begin{tabular}{|c|c|c|c|c|c|c|}
\hline Descripción & $\begin{array}{c}\text { Empresa } \\
\text { analizada }\end{array}$ & $\begin{array}{c}\text { Competidor } \\
01 \\
\end{array}$ & $\begin{array}{c}\text { Competidor } \\
02 \\
\end{array}$ & $\begin{array}{c}\text { Competidor } \\
03\end{array}$ & $\begin{array}{c}\text { Competidor } \\
04\end{array}$ & $\begin{array}{c}\text { Competidor } \\
05\end{array}$ \\
\hline Accesorios & $\mathrm{x}$ & & & $\mathrm{x}$ & & \\
\hline Alarmas & $\mathrm{x}$ & $\mathrm{x}$ & & $\mathrm{x}$ & $\mathrm{x}$ & $\mathrm{x}$ \\
\hline \multicolumn{7}{|l|}{ Aspirador portátil } \\
\hline Autoclaves & & $\mathrm{x}$ & & & & \\
\hline Bombas de vacío & $\mathrm{x}$ & $\mathrm{x}$ & & & & \\
\hline Brazos médicos & $\mathrm{x}$ & & & & & \\
\hline Cabeceros murales & $\mathrm{x}$ & & & & & $\mathrm{x}$ \\
\hline Caja de control de gases & $\mathrm{x}$ & $\mathrm{x}$ & & & & \\
\hline Cajas para seccionamiento & $\mathrm{x}$ & & & & & \\
\hline Canalización de gases & & & & & & $\mathrm{x}$ \\
\hline Canastas & $\mathrm{x}$ & $\mathrm{x}$ & & & & \\
\hline Carro multifuncional & $\mathrm{x}$ & & $\mathrm{x}$ & & & \\
\hline Central de vacío & $\mathrm{x}$ & & & & & $\mathrm{x}$ \\
\hline $\begin{array}{l}\text { Columnas articuladas, médi- } \\
\text { cas quirófanos }\end{array}$ & $\mathrm{x}$ & $\mathrm{x}$ & $\mathrm{x}$ & & $\mathrm{x}$ & $\mathrm{x}$ \\
\hline Compresores & & $\mathrm{x}$ & & & & $\mathrm{x}$ \\
\hline Consolas médicas & $\mathrm{x}$ & $\mathrm{x}$ & & & & \\
\hline Cortinas divisorias & & & & $\mathrm{x}$ & & \\
\hline Deslizador, accesorios & $\mathrm{x}$ & $\mathrm{x}$ & & & & \\
\hline Diseño de redes & & $\mathrm{x}$ & & & & \\
\hline
\end{tabular}




\begin{tabular}{|c|c|c|c|c|c|c|}
\hline Descripción & $\begin{array}{r}\text { Empresa } \\
\text { analizada }\end{array}$ & $\begin{array}{c}\text { Competidor } \\
01\end{array}$ & $\begin{array}{c}\text { Competidor } \\
02\end{array}$ & $\begin{array}{c}\text { Competidor } \\
03\end{array}$ & $\begin{array}{c}\text { Competidor } \\
04\end{array}$ & $\begin{array}{c}\text { Competidor } \\
05\end{array}$ \\
\hline $\begin{array}{l}\text { Equipos de cuidados } \\
\text { intensivos }\end{array}$ & & & & & $\mathrm{x}$ & \\
\hline \multicolumn{7}{|l|}{ Flujo, metros } \\
\hline Hermes seguridad & & & & & $\mathrm{x}$ & \\
\hline Lámparas de iluminación & $\mathrm{x}$ & & & & & \\
\hline Lámparas, quirófanos & $\mathrm{x}$ & & & & $\mathrm{x}$ & $\mathrm{x}$ \\
\hline Llamado de enfermería & $\mathrm{x}$ & $\mathrm{x}$ & & & & \\
\hline Mantenimiento & & $\mathrm{x}$ & $\mathrm{x}$ & & & \\
\hline Paneles & $\mathrm{x}$ & $\mathrm{x}$ & $\mathrm{x}$ & $\mathrm{x}$ & $\mathrm{x}$ & \\
\hline Pared hospitalaria & $\mathrm{x}$ & & & & & \\
\hline Planta de oxígeno & & & & & & $\mathrm{x}$ \\
\hline Porta líquidos & $\mathrm{x}$ & $\mathrm{x}$ & & & & \\
\hline Protectores de pared & & & & $\mathrm{x}$ & & \\
\hline \multicolumn{7}{|l|}{ Puntos para gases } \\
\hline $\begin{array}{l}\text { Regulación y control de } \\
\text { gases }\end{array}$ & $\mathrm{x}$ & & & $\mathrm{x}$ & & \\
\hline Reguladores & $\mathrm{x}$ & $\mathrm{x}$ & & & & \\
\hline Riel para accesorios & $\mathrm{x}$ & & $\mathrm{x}$ & & & \\
\hline Seguridad eléctrica & & & & & $\mathrm{x}$ & \\
\hline \multicolumn{7}{|l|}{ Servicio de inspección } \\
\hline $\begin{array}{l}\text { Sistema de brazos } \\
\text { monitorizados }\end{array}$ & $\mathrm{x}$ & & & & $\mathrm{x}$ & \\
\hline Sistema de rieles & $\mathrm{x}$ & & & & & $\mathrm{x}$ \\
\hline Sistemas de alta complejidad & & & & $\mathrm{x}$ & & \\
\hline Soporte bomba & $\mathrm{x}$ & $\mathrm{x}$ & & & & \\
\hline Soporte para monitor & $\mathrm{x}$ & & $\mathrm{x}$ & & & \\
\hline Soporte quirófanos & & & & & $\mathrm{x}$ & \\
\hline Superficie de apoyo & & $\mathrm{x}$ & & & & \\
\hline Tomas de gases & & & & & & $\mathrm{x}$ \\
\hline Total & 30 & 18 & 7 & 8 & 10 & 11 \\
\hline
\end{tabular}

Elaboración propia, 2020.

En la Tabla 2, se puede observar que existe similitud entre los productos que ofrece las empresas competidoras; con el competidor 01 , los productos que no comercializa la empresa analizada son aquellos que se consideran como dispositivos médicos y no son del interés particular de la empresa. 
La diferencia con el competidor 02 radica en que ellos comercializan materiales hospitalarios que no están dentro de la misión empresarial y que son considerados fáciles de comercializar por almacenes de cadena, como las cortinas divisorias. Respecto al competidor 03 , este es una compañía grande con buena trayectoria en el mercado y se caracteriza por la distribución de los equipos médicos a nivel mundial, comercializa los mismos productos de la empresa analizada, a diferencia de equipos eléctricos que no hacen parte de la razón de ser de la compañía y no se visualiza como un producto estrella para su distribución.

El competidor 03 es una empresa reconocida a nivel mundial por su especialidad en gases medicinales y son muy competitivos frente a este ramo, a pesar de ser considerados competencia por comercializar productos de arquitectura hospitalaria, la empresa estudiada ha tenido relaciones comerciales con esta empresa, dado que en un par de oportunidades se les ha vendido equipos que ellos no tienen como cabeceros pediátricos y que no están dentro de su interés comercializarlos en dicho país.

La diferencia entre los competidores 04 y 05 es que ellos comercializan productos que son dispositivos médicos y la compañía no tiene interés en este tipo de equipos.

\section{La amenaza de aparición de nuevos competidores}

En la actualidad no se tiene certeza que un nuevo competidor haga aparición en el mercado, puesto que se trata de un producto específico que necesita de varios años para poder llegar a ser certificado a nivel internacional y poder convertirse en una competencia directa, a nivel nacional los competidores directos están plenamente identificados y se tiene claridad sobre las líneas de productos que actualmente ofrecen en el mercado.

Tabla 3

Proveedores

\begin{tabular}{|c|c|c|c|}
\hline Proveedor & Producto & Exigencia al proveedor & $\begin{array}{l}\text { Exigencia del } \\
\text { proveedor }\end{array}$ \\
\hline \multirow[b]{3}{*}{$\begin{array}{l}\text { Provedores de } \\
\text { matérias primas e } \\
\text { insumos }\end{array}$} & Lamina. & \multirow{3}{*}{$\begin{array}{l}\text { La exigencia para todos } \\
\text { los proveedores es el } \\
\text { cumplimiento absoluto } \\
\text { de las condiciones en } \\
\text { cuanto a calidad, tiem- } \\
\text { pos de entrega y cum- } \\
\text { plimento de especifica- } \\
\text { ciones técnicas. }\end{array}$} & \multirow{3}{*}{$\begin{array}{l}\text { Cumplimiento en pagos, } \\
\text { constancia en compras, } \\
\text { información clara so- } \\
\text { bre las especificaciones } \\
\text { del servicio requerido. } \\
\text { Cumplimiento de ac- } \\
\text { uerdos de seguridad y } \\
\text { confidencialidad. }\end{array}$} \\
\hline & Tornillería. & & \\
\hline & $\begin{array}{l}\text { Componentes } \\
\text { Eléctricos. }\end{array}$ & & \\
\hline
\end{tabular}




\begin{tabular}{|c|c|c|c|}
\hline Proveedor & Producto & Exigencia al proveedor & $\begin{array}{l}\text { Exigencia del } \\
\text { proveedor }\end{array}$ \\
\hline \multirow[b]{3}{*}{$\begin{array}{l}\text { Proveedores de } \\
\text { servicios }\end{array}$} & Pintura electrostática. & \multirow{3}{*}{$\begin{array}{l}\text { La exigencia para todos } \\
\text { los proveedores es el } \\
\text { cumplimiento absoluto } \\
\text { de las condiciones en } \\
\text { cuanto a calidad, tiem- } \\
\text { pos de entrega y cum- } \\
\text { plimento de especifica- } \\
\text { ciones técnicas. }\end{array}$} & \multirow{3}{*}{$\begin{array}{l}\text { Cumplimiento en pagos, } \\
\text { constancia en compras, } \\
\text { información clara so- } \\
\text { bre las especificaciones } \\
\text { del servicio requerido. } \\
\text { Cumplimiento de ac- } \\
\text { uerdos de seguridad y } \\
\text { confidencialidad. }\end{array}$} \\
\hline & Galvanizado. & & \\
\hline & Soldadura. & & \\
\hline $\begin{array}{l}\text { Proveedores de } \\
\text { bienes y servicios } \\
\text { administrativos }\end{array}$ & Aduana. & $\begin{array}{l}\text { La exigencia para todos } \\
\text { los proveedores es el } \\
\text { cumplimiento absoluto } \\
\text { de las condiciones en } \\
\text { cuanto a calidad, tiem- } \\
\text { pos de entrega y cum- } \\
\text { plimento de especifica- } \\
\text { ciones técnicas. }\end{array}$ & $\begin{array}{l}\text { Cumplimiento en pagos, } \\
\text { constancia en compras, } \\
\text { información clara so- } \\
\text { bre las especificaciones } \\
\text { del servicio requerido. } \\
\text { Cumplimiento de ac- } \\
\text { uerdos de seguridad y } \\
\text { confidencialidad. }\end{array}$ \\
\hline
\end{tabular}

Elaboración propia, 2020.

Tanto para materias primas como para servicios se tienen múltiples proveedores que cumplen con las especificaciones técnicas que la empresa necesita para la fabricación de los equipos hospitalarios. Las exigencias hechas a todos son las mismas en cuanto al cumplimiento de todas las condiciones negociadas debido a los altos estándares de calidad que se necesitan para cumplir con los requisitos exigidos por la certificación UL. Lo anterior significa que el poder de negociación de los proveedores se limita a las condiciones de mercado, en la medida en que para ninguno de los bienes o servicios existe exclusividad.

\section{Amenaza de productos sustitutos}

Los equipos médicos que ofrece la empresa analizada no tienen un sustituto, debido a que son equipos de primera necesidad hospitalaria, los cuales por su especificidad deben cumplir con requisitos sanitarios que son imposibles de sustituir; se pueden hacer modificaciones tecnológicas que hagan del producto algo cada vez más sofisticado, cumpliendo con la seguridad de los pacientes y con la regulación sanitaria de cada país, cosa que en este momento el mercado no ofrece.

La tercera fase consistió en recopilar información de los tres países, Chile, Ecuador y Perú, realizando un análisis PEST, para poder tomar decisiones gerenciales frente a la incursión en el mercado internacional. 
Tabla 4

Análisis PEST

\begin{tabular}{|c|c|c|c|}
\hline Variable & Perú & Chile & Ecuador \\
\hline Política & $\begin{array}{l}\text { Según la encuesta hecha por } \\
\text { el Centro Estratégico Lati- } \\
\text { noamericano de Geopolítica } \\
\text { (CELAG) en } 2018 \text {, la mitad } \\
\text { de la población opina que } \\
\text { Perú atraviesa por una crisis } \\
\text { política, y el } 80 \% \text { de los en- } \\
\text { cuestados manifiestan tener } \\
\text { sentimientos negativos so- } \\
\text { bre la situación política. }\end{array}$ & $\begin{array}{l}\text { Posee un sistema político } \\
\text { estable y, en los últimos } \\
\text { años, se ha convertido } \\
\text { en un líder regional, } \\
\text { debido a su estabilidad } \\
\text { democrática y a que ga- } \\
\text { rantiza un ambiente se- } \\
\text { guro para hacer negocios. }\end{array}$ & $\begin{array}{l}\text { Ecuador es un país que vive una } \\
\text { profunda crisis de representación, } \\
\text { dada por la ausencia de posturas } \\
\text { fuertes e inspiradoras. Ni el go- } \\
\text { bierno, ni el correísmo, ni los ac- } \\
\text { tores que fueron de oposición han } \\
\text { logrado consolidarse como reales } \\
\text { opciones de gobierno. }\end{array}$ \\
\hline Económica & $\begin{array}{l}\text { Pese a las sucesivas crisis } \\
\text { políticas y escándalos de cor- } \\
\text { rupción que ha vivido el país, } \\
\text { la economía de Perú no ha } \\
\text { resultado tan afectada y, de } \\
\text { hecho, es una de las que más } \\
\text { crece en América Latina. }\end{array}$ & $\begin{array}{l}\text { Ha sido una economía } \\
\text { sólida en las últimas } \\
\text { décadas, debido a un } \\
\text { marco macroeconómico } \\
\text { estable que le ha per- } \\
\text { mitido amortiguar los } \\
\text { efectos de un contexto } \\
\text { internacional volátil y } \\
\text { reducir la pobreza. }\end{array}$ & $\begin{array}{l}\text { Iniciando el siglo, Ecuador exper- } \\
\text { imentó un período de crecimiento } \\
\text { económico y de reducción de la } \\
\text { pobreza. Sin embargo, algunos } \\
\text { problemas estructurales persist- } \\
\text { ieron como la expansión de un } \\
\text { sector público poco eficiente, } \\
\text { desbalances macroeconómicos } \\
\text { importantes, carencia de mecanis- } \\
\text { mos de estabilización y una limit- } \\
\text { ada inversión del sector privado. }\end{array}$ \\
\hline Social & $\begin{array}{l}\text { Actualmente, las condi- } \\
\text { ciones externas demandan } \\
\text { reformas complementarias } \\
\text { que eleven la productividad } \\
\text { y promuevan la prosperidad } \\
\text { compartida. En los últimos } \\
\text { tres años no ha sido posible } \\
\text { la implementación de esas } \\
\text { reformas debido a la gran } \\
\text { inestabilidad política y so- } \\
\text { cial, que ha llevado al país } \\
\text { a tener tres presidentes en } \\
\text { tres años. }\end{array}$ & $\begin{array}{l}\text { Chile tuvo la democra- } \\
\text { cia ininterrumpida más } \\
\text { larga del continente, an- } \\
\text { tes del golpe de Estado } \\
\text { que instaló la dictadura } \\
\text { de Augusto Pinochet en } \\
\text { 1973, y ha disfrutado de } \\
\text { una democracia ininter- } \\
\text { rumpida desde la caída } \\
\text { pacífica de su régimen, } \\
\text { en } 1990 \text {. Esto ha hecho } \\
\text { que Chile sea la nación } \\
\text { más estable y próspera } \\
\text { de América Latina. }\end{array}$ & $\begin{array}{l}\text { El Gobierno de Lenín Moreno } \\
\text { enfrenta una fuerte protesta so- } \\
\text { cial en todo el país, desde que } \\
\text { anunciara el pasado mes de oc- } \\
\text { tubre (2019) una serie de medidas } \\
\text { económicas que sus detractores } \\
\text { denominan "el paquetazo". Esta } \\
\text { situación ha llevado a la misma } \\
\text { ONU y a la CIDH a expresar su } \\
\text { preocupación por la proclama- } \\
\text { ción del Estado de excepción en } \\
\text { ese país, ante las fuertes protestas } \\
\text { en varias ciudades contra las me- } \\
\text { didas económicas. }\end{array}$ \\
\hline $\begin{array}{l}\text { Tecnológi- } \\
\text { ca }\end{array}$ & $\begin{array}{l}\text { En los últimos años, las tec- } \\
\text { nologías de información se } \\
\text { han incorporado con más } \\
\text { fuerza a las empresas peru- } \\
\text { anas, tanto en las pequeñas } \\
\text { y medianas como en las } \\
\text { grandes. Uno de los grandes } \\
\text { temas que favorece este pro- } \\
\text { ceso es un gran concepto } \\
\text { que se está dando y promov- } \\
\text { iendo en Perú: la transfor- } \\
\text { mación digital. }\end{array}$ & $\begin{array}{l}\text { Chile ha introducido un } \\
\text { índice de desarrollo dig- } \\
\text { ital para medir avances } \\
\text { tecnológicos. Es el país } \\
\text { con mejor desarrollo tec- } \\
\text { nológico en Sudamérica } \\
\text { y conserva la tercera } \\
\text { posición entre las } 25 \\
\text { economías emergentes } \\
\text { del mundo y la primera } \\
\text { de América Latina. }\end{array}$ & $\begin{array}{l}\text { En } 2019 \text {, Ecuador se ubicó en el } \\
\text { puesto } 97 \text { en el Global Innovation } \\
\text { Index. }\end{array}$ \\
\hline
\end{tabular}

Elaboración propia, 2020. 


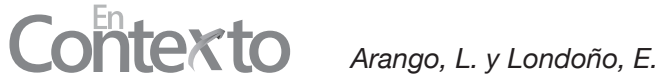

\section{Discusión}

Para el desarrollo de esta investigación fue significativo analizar temas fundamentales para tomar decisiones con respecto al posicionamiento de la marca para Bioinnova S.A.S., uno de ellos fue la importancia que tiene la diferenciación del producto, mediante "la distinción entre productos que cumplen el mismo propósito, pero fueron hechos por diferentes productores y además, compiten entre ellos" (Kotler, 1994). Esta definición adopta un enfoque que incluye todos los aspectos de la oferta y, además, asegura que diferenciación significa la introducción de características diferenciales, bien sea de calidad, estilo o imagen de la marca, como base para ser líder en el mercado; dichas características tendrán que cumplir con ciertos requisitos, que deberán ser percibidas por los consumidores, no podrán presentar dudas con respecto a posibles imitaciones de la competencia y tendrán un valor real para el proveedor (Cardozo et al., 2014).

La diferenciación es una estrategia de marketing que trata de resaltar las características del producto, sustanciales o accesorias, que pueden contribuir a que se perciba como único, para así crear una situación de monopolio en el mercado, pero esta diferenciación contendrá atributos esperados por el consumidor y atributos complementarios de los productos (Kotler, 1994).

Los atributos esperados, aunque en realidad no son absolutamente necesarios, el comprador los espera, razón por la cual, siempre deberán estar incluidos en la oferta del producto, en caso contrario, el consumidor optará por otra opción que sí los incorpore, es decir, la oferta del producto puede ser excluyente, no por incluir ciertos atributos, sino por no incluirlos.

Las empresas modernas deben asumir una estrategia general que se enfoque exactamente en la creación de una posición estratégica única y valiosa para la empresa. Se trata de una posición competitiva que deberá crear diferencias reales con las demás empresas de su sector. De acuerdo con Porter (1985), las empresas podrán optar por tres diferentes rutas que les garanticen, a futuro, una mejor posición frente a la competencia:

1. El primer camino se refiere a una estrategia genérica de costos. Se trata de una estrategia que ha venido siendo aplicada en la gran mayoría de las empresas nacionales y que se desarrolla con el propósito de lograr eficiencia en los procesos y concebir economías de escala que permitan ofrecer a sus clientes un producto con un costo más bajo. 
2. En la segunda vía, se opta por una estrategia genérica de enfoque, la cual se aplica puntualmente sobre un nicho de mercado específico, dependiendo de las necesidades propias de este grupo de clientes.

3. El último camino, aunque no es tan empleado como los anteriores, constituye una oportunidad, porque se trata de una estrategia genérica de diferenciación, la cual presenta como característica esencial ofrecer a los clientes productos con valor superior en lo que se refiere al diseño del producto, a su funcionalidad y su servicio.

El posicionamiento de la marca en marketing, se refiere al lugar que ocupa esta en la mente de los consumidores con respecto de sus competidores. La marca es un signo distintivo que identifica los productos o servicios de una empresa o empresario permitiendo a los consumidores identificar un producto o servicio y recordarlo de forma que puedan diferenciarlo de uno igual o semejante ofrecido por otra empresa.

Para la Asociación Americana de Marketing, marca es un nombre, un término, una señal, un símbolo, un diseño o la combinación de algunos de estos significados que identifica productos y servicios de una empresa y la diferencia de los competidores. Así, la marca es el nombre, término, logo, diseño, símbolo o una combinación integrada de ellos que tiene como propósito identificar y distinguir un producto o servicio de los otros de su misma especie y que representa un vínculo, que puede llegar a ser bastante fuerte y determinante entre la oferta y el consumidor.

Ahora, el posicionamiento es reconocido como un término relativamente reciente, que creció y evolucionó de las discusiones de segmentación de los mercados, del público-objetivo y de las estructuras de marketing entre las décadas de los 60's y 70's. El concepto de posicionamiento apareció en la industria de bienes de consumo masivo con el posicionamiento del producto (Trout, 2002). Sin embargo, existe otra hipótesis, según la cual es la evolución del posicionamiento a partir de las medidas de las percepciones de los consumidores y de los mapas perceptuales (Serralvo y Tadeu, 2005).

El posicionamiento es aquello que una marca está dispuesta a hacer para ser quien quiere ser. Delimita el espacio que ocupará una marca en sus mercados. Esto significa que es una herramienta fundamental que determina quién es, qué hace y por qué es importante para su mercado. Asimismo, determina el significado y la forma en la que se va a competir en ese mercado; consecuentemente, el posicionamiento de la marca señala qué espacio ocupa la marca en la mente de los consumidores. 


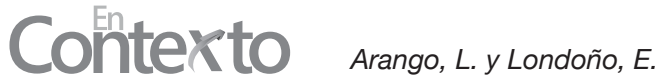

Además, la investigación de los mercados ha acelerado en gran medida los procesos de internacionalización de las empresas a nivel mundial. En este contexto, la exportación se presenta como la vía más utilizada y tradicional que utilizan las empresas para su entrada en los mercados foráneos, configurándose como la opción estratégica más efectiva para asegurar la supervivencia y el crecimiento de las organizaciones que han optado por iniciar sus propios procesos de internacionalización. Las empresas ven en las exportaciones el medio directo para hacer frente a la competencia extranjera en sus mercados nacionales, así como la forma de ampliar su cobertura en el mercado y, por esta vía, aumentar su rentabilidad. No obstante, las exportaciones como tales no son un fin en sí mismo, puesto que el objetivo de las empresas exportadoras será obtener ventajas competitivas en los países y/o mercados donde pretendan competir (Delgado-Márquez et al., 2013).

La investigación de mercados no es más que el resultado de la convergencia de la evolución de la inteligencia competitiva con la emergencia del mercadeo como un área estratégica de la empresa. Una investigación de mercados, necesariamente deberá tener una visión clara y exacta que le permita obtener unos resultados esperados por las empresas. Ahora, fundamentándose en el estudio de Merino et al. (1991), se puede decir que una investigación de mercados tiene tres objetivos fundamentales que son el social, económico y administrativo.

Los objetivos específicos de esta investigación se cumplieron mediante el resultado de un análisis de las condiciones del mercado para llevar a cabo el proceso de posicionamiento de la marca en Chile, Ecuador y Perú, lo cual permitió tomar la decisión de seleccionar a Chile, debido a múltiples factores, dentro de los que se cuentan principalmente la estabilidad y la fortaleza de todos los medidores macroeconómicos, al crecimiento de la inversión extranjera directa, a las perspectivas de crecimiento y al alto poder adquisitivo de sus ciudadanos, que colocan a Chile entre los dos primeros países Latinoamericanos en ese medidor. Estos aspectos suponen una reducción importante del riesgo en los negocios que se desarrollen en ese país.

También se tuvo en cuenta la estabilidad de una democracia que ya ha cumplido 30 años desde la caída de la dictadura, pasando por gobiernos de derecha e izquierda, pero manteniendo un fuerte principio democrático, lo que se traduce en un ambiente de seguridad en el desarrollo de relaciones comerciales de largo plazo. En efecto, Chile es un país que invierte alrededor del 7,2\% del PIB en salud, que es un dato importante, si se tiene en cuenta el monto del PIB (US \$ 298.200 millones - Banco Mundial) y la población del país (18.9 millones de habitantes - Datos INE, 2019). 
Se identificaron los requisitos para el registro de la marca en cada uno de los países citados con el fin de:

- Crear un activo intangible para la empresa.

- Generar diferenciadores de competencia.

- Proteger el activo a través del registro.

Este registro se hizo para Colombia y Chile, a través de una empresa de asesoría jurídica llamada Singular, la cual hace los trámites necesarios ante la Superintendencia de Industria y Comercio, dicho proceso tarda entre 8 y 12 meses. El registro se comenzó el 16 de Julio del 2019, y en la actualidad Bioinnova S.A.S. aún se encuentra en espera de la respuesta por parte de la entidad encargada, con actualizaciones frecuentes por parte del apoderado.

Antes de comenzar con esta investigación el organigrama de la compañía estaba conformado por 16 cargos, siendo la administración responsable del funcionamiento general de la compañía, respaldada por la gerencia, además de cumplir con funciones que podrían ser delegadas a otras personas con las competencias necesarias y el tiempo suficiente para desarrollar su labor.

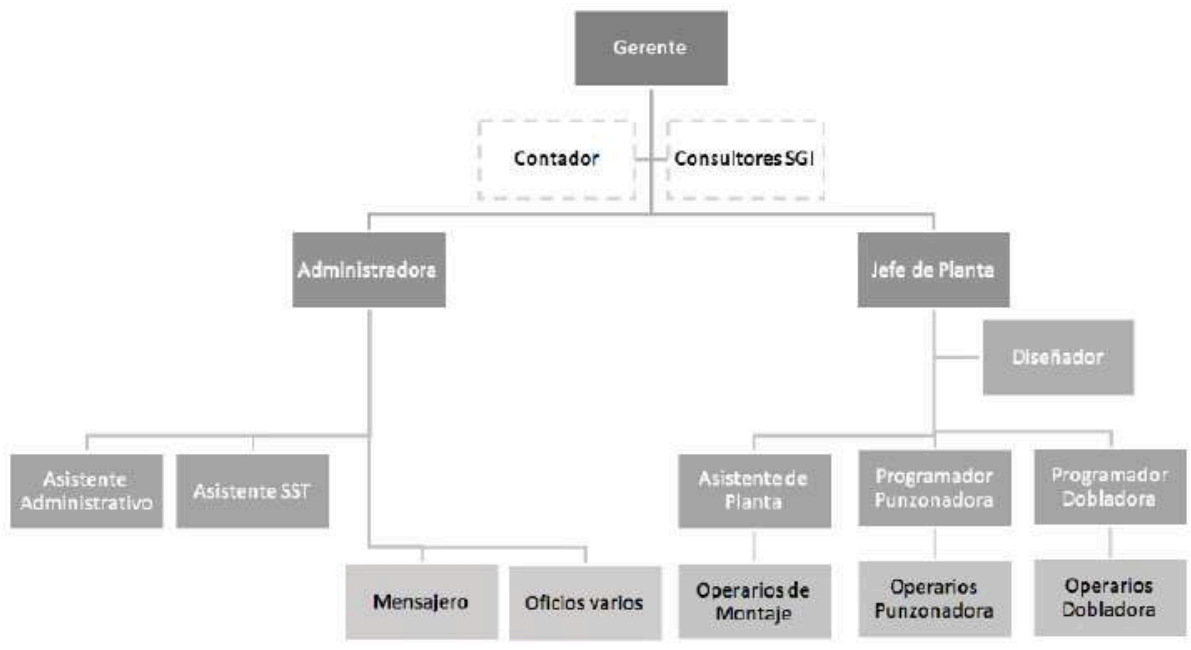

Figura 3. Organigrama 2019

Bioinnova S.A.S.

Con el paso del tiempo, se lograron establecer cambios en la estructura organizacional de Bioinnova S.A.S., aumentando el grupo de trabajo con el fin 


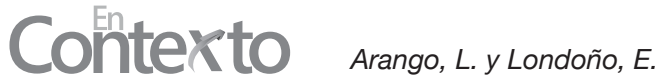

de ingresar a nuevos mercados y liderar el proceso de comercio exterior; estas personas tienen la función de:

- Confirmar con producción si se cuenta con la capacidad suficiente para cumplir con el pedido del cliente.

- Verificar con calidad que los productos cuenten con las especificaciones técnicas solicitadas por el cliente.

- Proceder con la puesta en marcha de la exportación, después de la previa autorización por parte de la administración de Bioinnova S.A.S.

- Encargarse de la contratación con los diferentes actores intermediarios de las operaciones de comercio exterior.

- Coordinar fechas de embarque, cumplir con los requisitos de transporte internacional y trámites aduaneros para la posterior salida de los productos.

- Estar al tanto del cumplimiento de las obligaciones cambiarias.

- Monitorear todo el proceso desde la salida de la empresa hasta la llegada a puerto o aeropuerto seleccionado por el cliente.

- Realizar seguimiento postventa y evaluar la satisfacción del cliente.

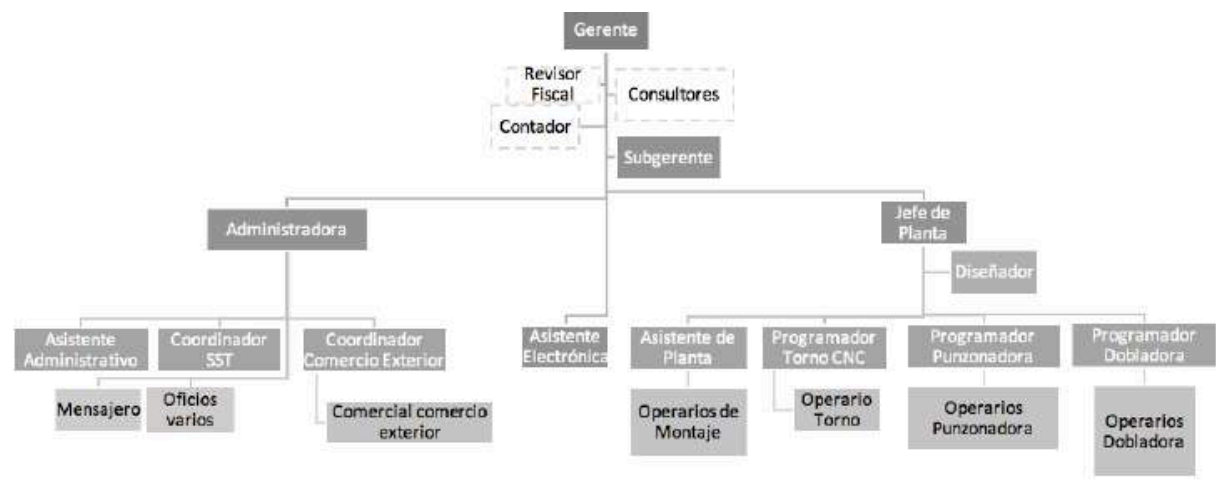

Figura 4. Organigrama 2020

Bioinnova S.A.S.

Además de incrementar el personal de la compañía a nivel administrativo, se compraron activos fijos que han sido representativos para agilizar procesos de producción.

Este cambio se hizo basado en el llamado diagnóstico potencial de internacionalización que se realizó a través del modelo de las cinco fuerzas de Porter (1985). 
El poder de negociación de los clientes: se seleccionaron los principales clientes de Bioinnova S.A.S. en Chile, Ecuador y Perú; luego, se hizo énfasis en los requerimientos que estos hacen a la compañía acerca de las características que son fundamentales para los productos. Todos coincidieron en calidad, productos funcionalmente confiables y seguros para los pacientes, diseño exclusivo, productos con estética acorde a la infraestructura hospitalaria, cumplimiento en las fechas de entrega, relación costo-beneficio, cumplimiento de contratos y confidencialidad.

Las exigencias de los clientes están plenamente identificadas por Bioinnova S.A.S., razón por la cual cumplir con estos requisitos, no solo satisface las necesidades y expectativas de los mismos, sino que también es la garantía para permanecer vigente en el mercado y ser un referente importante para nuevos clientes.

Competencia en el mercado: después de realizado un análisis de la competencia, basado en la similitud de los productos comercializados, se puede aseverar que:

- Existen pocas empresas a nivel nacional e internacional que fabriquen y distribuyan esta clase de productos al sector.

- Si hay una gran semejanza entre los productos, debido a que el sector lo exige y deben cumplir normas sanitarias en todos los países en pro de la seguridad del paciente.

- El mercado es bastante amplio y la oferta es poca.

- Bioinnova S.A.S. entró a competir con empresas que tienen amplia trayectoria en el mercado y ha logrado penetrar el sector de forma satisfactoria.

La amenaza de aparición de nuevos competidores: en la actualidad no se tiene certeza que un nuevo competidor haga aparición en el mercado, puesto que se trata de un producto específico que necesita de varios años para poder llegar a ser certificado a nivel internacional y poder convertirse en una competencia directa.

El poder de negociación de los proveedores: Bioinnova S.A.S. cuenta con proveedores de materia prima, servicios administrativos y logísticos. La consecución de estos proveedores tiene un procedimiento especial por regulaciones establecidas por el sistema de gestión de calidad, dado que estos deben cumplir con requisitos específicos que son fundamentales para que el producto final satisfaga las necesidades del cliente.

Se ha logrado un vínculo interesante donde se pueden enlazar las políticas empresariales, logrando beneficios para todos. Siempre y cuando se respeten las condiciones iniciales de trabajo la relación cliente-proveedor, se sostendrá en el tiempo. 


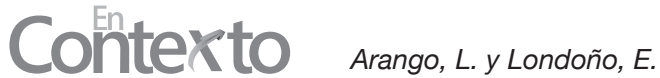

Amenaza de productos sustitutos: los equipos médicos que ofrece Bioinnova S.A.S. no tienen un sustituto, debido a que son equipos de primera necesidad hospitalaria, los cuales por su especificidad deben cumplir con requisitos sanitarios que son imposibles de sustituir, estos productos sí se pueden modificar tecnológicamente, convirtiéndolos en más sofisticados y novedosos, pero cumpliendo siempre con la seguridad de los pacientes y con la regulación sanitaria de cada país, cosa que en este momento el mercado no ofrece.

\section{Conclusiones}

Analizar la empresa mediante las cinco fuerzas de Porter (1985), permitió conocer las fortalezas, oportunidades, amenazas y debilidades de Bioinnova S.A.S., lo cual llevó al desarrollo de una investigación cualitativa-descriptiva, basándose en fuentes secundarias: como información recolectada en libros, revistas especializadas, anuarios e internet. Adicionalmente, se realizó una encuesta de satisfacción mediante Google Forms a 10 clientes internacionales, la cual permitió conocer el índice de cumplimiento de las especificaciones técnicas requeridas por ellos, identificar qué concepto tienen sobre la calidad, servicio ofrecido y el cumplimiento de las fechas de entrega.

El resultado de esta encuesta fue importante para la toma de decisiones sobre la internacionalización, a través del posicionamiento de la marca. Para esto, se identificaron los tres principales países donde se visualizó una oportunidad de negocio importante, se hizo análisis PEST, sobre Chile, Ecuador y Perú, el cual permitió seleccionar a Chile, por ser uno de los países que más invierte en salud.

Se posicionará la marca mediante la implementación de estrategias de diferenciación de productos, aprovechando las dos principales ventajas que tiene Bioinnova S.A.S.: calidad y servicio. Con el fin de lograr posicionamiento y garantizar la creación de valor para Bioinnova S.A.S., se hizo un registro de la marca que tarda entre 8 y 12 meses. Este proceso se le asignó a un apoderado en junio de 2019, sin obtener respuesta hasta el momento por parte de las Superintendencias de Industria y Comercio, tanto de Colombia como de Chile.

En el desarrollo de esta investigación, la cual se realizó entre octubre de 2018 a noviembre de 2019, se tuvieron pocas limitaciones, dado que el personal de la empresa estuvo dispuesto todo el tiempo para la obtención de información relevante. La empresa sigue en la implementación de las directrices determinadas por la investigación sobre el cumplimiento de los objetivos de este proyecto, con adelantos significativos. Para el proceso de posicionamiento de marca se hicieron cambios organizacionales, como la contratación de personal administrativo con el fin de liderar el proceso de comercio exterior. 


\section{Referencias}

Arango, L. (2019). Encuesta de Satisfacción Bioinnova S.A.S. Bioinnova S.A.S.

Cardozo, J., Hernandis, B., y Ramírez, N. (2014). Caracterización de los sistemas de productos en el marco de la personalización, la variabilidad y la diferenciación. Un estudio con expertos. Revista chilena de ingeniería, 22(2), 278-291.

Delgado-Márquez, B., Escudero-Torres, M., \& Hurtado-Torres, N. (2013). Internationalised strengthens your reputation: an empirical investigation of top higher education institutions. Higher Education, 66, 619-633.

Godás, L. (2006). El producto. Tipos, atributos y diferenciación. Gestión farmacéutica, 25(5), 116-120.

Kotler, P. (1994). Dirección de mercadotecnia. Prentice Hall Hispanoamericana.

Medina-Aguerrebere, P. (2014). Marca y comunicación empresarial. Editorial UOC.

Merino, M., Pintado, T., Sánchez, J., y Grande, I. (1991). Introducción a la investigación de mercados. McGraw Hill.

Ortegón, L. (2014). Gestión de marca. Conceptualización, diseño, registro, construcción y evaluación. Politécnico GranColombiano.

Porter, M. (1985). Ventaja competitiva. First Free Press Edition.

Review, H. (2000). Gestión de marcas. Deusto.

Serralvo, F., y Tadeu, M.(2005). Tipologias del posicionamiento de marca. Un estudio conceptual en Brasil y en España. Revista galega de economía, 14(1-2), 1-5.

Trout, R. (2002). Posicionamiento una batalla por su mente. Makron Books.

Zapata, G. (2019). El determinismo en la teoría contingente. Orígenes de sus fundamentos teóricos para su comprensión. Ciencias y sociedad, 39(1), 121-154.

\section{Para citar este artículo:}

Arango, L. y Londoño, E. (2021). Análisis del posicionamiento de las marcas de equipos hospitalarias. Caso de estudio empresa Bioinnova S.A.S. En-Contexto, 9(14), 85-105. 